\title{
Personalized News Video Recommendation
}

\author{
Hangzai Luo ${ }^{1}$, Jianping Fan ${ }^{1}$, Daniel A. Keim ${ }^{2}$, and Shin'ichi Satoh ${ }^{3}$ \\ 1 CS Department, UNC-Charlotte, USA, hluo, jfan@uncc.edu \\ 2 University of Konstanz, keim@uni-konstanz.de \\ ${ }^{3}$ NII, Tokyo, Japan, satoh@nii.ac.jp
}

\begin{abstract}
In this paper, a novel framework is developed to support personalized news video recommendation. First, multi-modal information sources for news videos are seamlessly integrated and synchronized to achieve more reliable news topic detection, and the contexts between different news topics are extracted automatically. Second, topic network and hyperbolic visualization are seamlessly integrated to support interactive navigation and exploration of large-scale collections of news videos at the topic level, so that users can gain deep insights of large-scale collections of news videos at the first glance. In such interactive topic network navigation and exploration process, users' personal background knowledge can be exploited for selecting news topics of interest interactively, building up their mental models of news needs precisely and formulating their queries easily by selecting the visible news topics on the topic network directly. Our system can further recommend the relevant web news, the new search directions, and the most relevant news videos according to their importance and representativeness scores. Our experiments on large-scale collections of news videos have provided very positive results.
\end{abstract}

Key words: Topic network, personalized news video recommendation.

\section{Introduction}

There are more than 30,000 television stations in the world, these television stations broadcast large amounts of TV news programs (news videos) every day. Due to the large number of broadcast channels and TV news programs, finding news videos of interest is not a trivial task: (a) Most existing content-based video retrieval (CBVR) systems assume that users can formulate their information needs precisely either in terms of keywords or example videos. Unfortunately, users may not be able to know what is happening now (i.e., if they know it, it is not a news), thus it is very hard for them to find the suitable keywords or example videos to formulate their news needs precisely without obtaining sufficient knowledge of the available news topics of interest. Thus there is an urgent need to develop new techniques for detecting news topics of interest from large-scale collections of news videos to assist users on finding news videos of interest more effectively. (b) Because the same news topic can be discussed in many TV channels and news programs, topic-based news search may return large amount of news videos and thus simple news search via topics may bring the serious problem of information overload to the users. (c) Most existing CBVR systems treat all 
users equally while completely ignoring the diversity and rapid change of their search interests. Besides the rapid growth of broadcast TV channels and news programs, we have also observed different scenarios of news needs from different users, thus it is very difficult to come up with a one size fits all approach for accessing large-scale collections of news videos. (d) The keywords for news topic interpretation may not be expressive enough for describing the rich details of video contents precisely and using only the keywords may not be able to capture users' search intentions effectively. Thus visualization is becoming a critical component of personalized news video recommendation system [3-8]. (e) The objectives for personalized video recommendation and contentbased video retrieval are very different, which make it unsuitable to directly apply the existing CBVR techniques for supporting personalized video recommendation. Thus supporting personalized news video recommendation is becoming one important feature of news services [1].

In this paper, we have developed a novel framework to support personalized news video recommendation, and our framework is significantly different from other existing works: (a) Rather than performing semantic video classification and automatic video content understanding on the single-modal channel of news videos, we have seamlessly integrated multi-modal information channels (audio, video and closed captions) to achieve more reliable news topic detection. (b) The associations among the news topics (i.e., inter-topic contexts) are determined automatically and an interestingness score is automatically assigned to each news topic via statistical analysis. Such interestingness scores are further used to select the news topics of interest and filter out the less interesting news topics automatically. (c) A hyperbolic visualization tool is incorporated to enable interactive topic network exploration and allow users to gain deep insights of large-scale collections of news topics at the first glance, so that they can make better search decisions and find the news topics of interest interactively according to their personal preferences. The user's personal knowledge, search intentions and contexts, which are disclosed and captured in the interactive topic network navigation and exploration process, can be taken into consideration for personalizing the topic network and the search results. (d) A novel video ranking algorithm is developed for recommending the relevant web news, the new search directions and the most relevant news videos according to their importance and representativeness scores for a given news topic.

\section{Related Work}

The Informedia Digital Video Library project at CMU has achieved significant progresses on analyzing, indexing and searching of large-scale collections of news videos [11-12], and several applications have been reported, such as semantic video understanding, multi-modal decision fusion, keyword-based video retrieval and query result visualization. Unfortunately, automatic video understanding is still an open problem for computer vision [7-8].

Visualization is widely used to help the users explore large amount of data collections and find interesting parts interactively [3-8]. In-spire [3] has been developed for visualizing and exploring large-scale text document collections, where statistics of news reports is put on a world map to inform the audiences of the "hotness" of regions and the relations among the regions. One major problem for such geographical location- 
based visualization approach is that some hot regions (such as middle-east) may be too busy but other places may be empty. TimeMine [4] is proposed to detect the most important reports and organize them through the timeline with the statistical models of the word usage. Another system, called newsmap [5], can organize news topics from Google news on a rectangle, where each news story covers a visualization space that is proportional to the number of related news pages reported by Google. News titles are drawn in the visualization space allocated to the relevant news topic.

It is very attractive to visualize the news topics according to their importance scores, but another aspect of news topics (i.e., inter-topic contexts) are missed by all these existing techniques (e.g., this could be a serious problem for all these existing techniques because the news topics of inetrest may never happen individually). Context between the news topics is also very important for users to make better search decisions, especially when the users are not familiar with the available news topics and their search goals or ideas are still fuzzy. The inter-topic context can give a good approximation of the interestingness of the news topics (i.e., like PageRank for characterizing the importance of web pages). Thus it is very attractive to integrate topic network (i.e., news topics and their inter-topic contexts) for characterizing the interestingness of the news topics, assisting users on making better search decisions and suggesting the future search directions.

ThemeRiver [6] can visualize large-scale collections of news documents with the keywords or the themes. ThemeRiver can intuitively represent the distribution structure of the themes and the keywords in the collections. Such distribution structures of the themes and the keywords may be useful for disclosing statistical knowledge of largescale collections of news documents, but they may not make any sense to the users according to their goals of news search because there is an interest gap between the distribution structures and the users' real news needs (e.g., news analysts may care such statistical knowledge, but general users as the news seekers may just care the news topics of interest and their inter-topic contexts).

When large-scale news collections come into view, the number of the available news topics could be very large and displaying all of them to the users may mislead them. To visualize the news topics of interest in a size-limited screen, most existing algorithms have to select the most significant news topics of interest according to their definitions of importance. Unfortunately, the importance score of a news topic may depend on two issues: (a) the relative importance of the given news topic in the pool of large-scale news video collections; (b) the personal preferences for each particular user which may not be known at the beginning. Thus selecting and disclosing only the most important news topics of interest according to the pre-defined criteria may hinder users to access some news topics, which are strongly related with their personal preferences but may not be significant in the large-scale news video collections. For example, the news topics for NBA playoff may be very interesting for basketball fans, but the news topics for president candidate ellection may always dominate the pool of news topics and have higher interestingness scores in general. Thus there is an urgent need to develop new algorithms which are able to take the user's personal preferences into consideration for defining the personalized interestingness scores of the news topics. 
There are two well-accepted approaches for supporting personalized information retrieval [9-10]: content-based filtering and collaborative filtering. Unfortunately, all these existing personalized information recommendation techniques largely depend on the collections of users' profiles and the available text context descriptions, thus they cannot support new users effectively because their profiles may not be available. Because of the shortage of the available text descriptions of news videos, all these existing techniques cannot directly be extended for enabling personalized news video recommendation and there is an urgent need to develop news frameworks.

\section{Personalized News Topic Recommendation}

In this paper, an interactive approach is developed by incorporating topic network and hyperbolic visualization to recommend the news topics of interest for assisting users on accessing large-scale collections of news videos more effectively. To do this, an automatic scheme is first developed to construct the topic network for representing and interpreting large-scale collections of news videos at the topic level. In addition, a hyperbolic visualization technique is integrated to enable interactive navigation and exploration of large-scale topic network and recommend the news topics of interest according to the users' personal preferences.

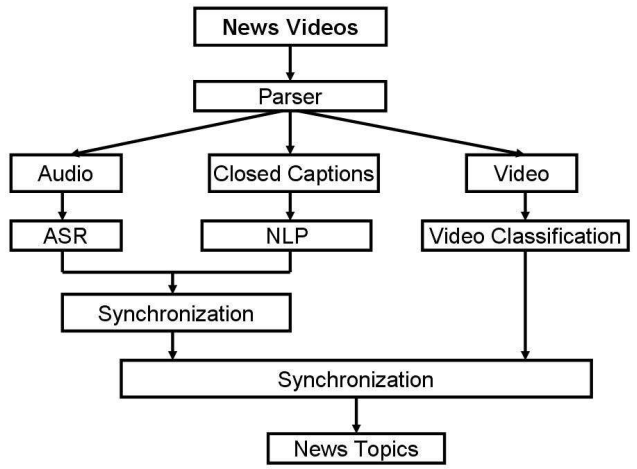

Fig. 1. The flowchart for synchronizing multiple sources for news topic detection.

Topic detection is important because many people often want to identify some specific news stories or topics that are relevant to their personal interests. For the TV news programs, there are three multi-modal information channels (audio, video and closed captions) that can be integrated and be synchronized to enable more reliable news topic detection. Because the inherent property of speech is purely semantic, lexical search from automatic speech recognition (ASR) transcripts has been treated as the most successful strategy for content-based video retrieval. The problem with the ASR transcripts is that the spoken words may not completely cover the abundant semantic contents available in the news video.

As shown in Fig. 1, we have developed a new scheme for automatic news topic detection by taking the advantage of multi-modal information channels (cross-media). First, automatic speech recognition (ASR), natural language processing (NLP), and semantic video classification are performed parallelly on these three multi-modal information channels to determime the keywords for news topic description from both the 
audio channel and the closed captions and detect the video concepts from the video channel. Second, the audio channel is synchronized with the closed captions, and the video channel is further synchronized with the audio channel and the closed captions. Finally, the news topic detection results from these three multi-modal information channels are integrated to boost the performance of our news topic detection algorithm. After the closed captions are synchronized with the news videos, we can assign the video shots to the most relevant news topics that are accurately detected from the closed captions. Thus all the video shots, which locate between the start time and the end time of a given new topic (that has been detected from the closed captions), are assigned to the given news topic automatically. Integrating multi-modal information channels for news topic detection can significantly enhance the detection accuracy.

The inter-topic contextual relationships are obtained automatically, where both the cosine similarity and the mutual similarity for the relevant news topics are used to define a new measurement for determining their inter-topic associations more precisely. The inter-topic context $\Upsilon\left(C_{i}, C_{j}\right)$ between two news topics $C_{j}$ and $C_{i}$ is determined by:

$$
\Upsilon\left(C_{i}, C_{j}\right)=\alpha \cdot \Phi\left(C_{i}, C_{j}\right)+\beta \cdot \Psi\left(C_{i}, C_{j}\right), \quad \alpha+\beta=1
$$

where the first part $\Phi\left(C_{i}, C_{j}\right)$ denotes the cosine similarity between the term weights for the text terms to interpret the news topics $C_{j}$ and $C_{i}$, the second part $\Psi\left(C_{i}, C_{j}\right)$ indicates the mutual similarity between the text terms for interpreting the news topics $C_{j}$ and $C_{i}$ according to their co-occurrence probability, $\alpha$ and $\beta$ are their relative importances.

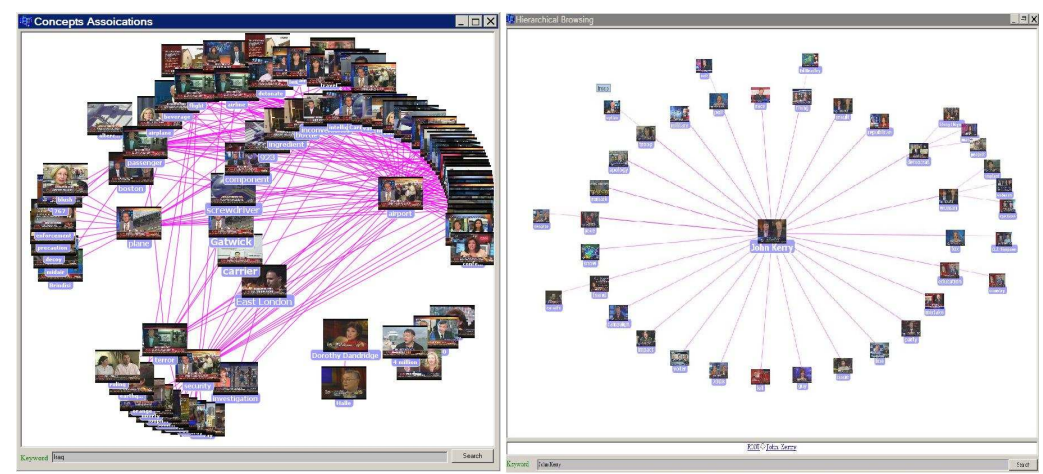

Fig. 2. Our large-scale topic network for organizing large-scale news videos.

The cosine similarity $\Phi\left(C_{i}, C_{j}\right)$ between the text terms for interpreting the news topics $C_{j}$ and $C_{i}$ can be defined as:

$$
\Phi\left(C_{i}, C_{j}\right)=\frac{\sum_{l=1}^{N} w_{l}\left(C_{i}\right) \cdot w_{l}\left(C_{j}\right)}{\sqrt{\sum_{l=1}^{N} w_{l}\left(C_{i}\right)^{2}} \sqrt{\sum_{l=1}^{N} w_{l}\left(C_{j}\right)^{2}}}
$$

where $N$ is the number of news documents (closed caption documents) in our collections, $w_{l}\left(C_{i}\right)$ and $w_{l}\left(C_{j}\right)$ are the weights for the text terms for interpreting the news 
topics $C_{j}$ and $C_{i}$.

$$
\left\{\begin{array}{l}
w_{l}\left(C_{i}\right)=\log \left(f_{l}\left(C_{i}\right)+1.0\right) \cdot \log \frac{N+1}{f_{l}+0.5} \\
w_{l}\left(C_{j}\right)=\log \left(f_{l}\left(C_{j}\right)+1.0\right) \cdot \log \frac{N+1}{f_{l}+0.5}
\end{array}\right.
$$

where $f_{l}\left(C_{i}\right)$ and $f_{l}\left(C_{j}\right)$ are the frequencies of the text terms for interpreting the news topics $C_{j}$ and $C_{i}$ in the $l$ th news document (closed caption document), $f_{l}$ is the frequency of the $l$ th news document which the news topics $C_{j}$ and $C_{i}$ occur in. The higher value of the cosine similarity $\Phi\left(C_{i}, C_{j}\right)$ implies the stronger association $\Upsilon\left(C_{i}, C_{j}\right)$ between the relevant news topics $C_{j}$ and $C_{i}$.

The mutual similarity $\Psi\left(C_{i}, C_{j}\right)$ between the news topics $C_{j}$ and $C_{i}$ can be defined as:

$$
\Psi\left(C_{i}, C_{j}\right)=\log \frac{p\left(C_{i}, C_{j}\right)}{p\left(C_{i}\right) \cdot p\left(C_{j}\right)}
$$

where $p\left(C_{i}, C_{j}\right)$ is the co-ocurrence probability of the relevant news topics $C_{j}$ and $C_{i}$ in the corpus, $p\left(C_{i}\right)$ and $p\left(C_{j}\right)$ are the individual ocurrence probability of the news topics $C_{j}$ and $C_{i}$ in the corpus. The underlying assumption behind the mutual similarity measurement $\Psi\left(C_{i}, C_{j}\right)$ is that two news topics co-occur frequently if they are strongly relevant. The higher value of the mutual similarity $\Psi\left(C_{i}, C_{j}\right)$ implies the stronger contexts $\Upsilon\left(C_{i}, C_{j}\right)$ between the relevant news topics $C_{j}$ and $C_{i}$.

Thus each news topic is automatically linked with multiple relevant news topics with the higher values of the inter-topic contexts $\Upsilon(\cdot, \cdot)$. A portion of our large-scale topic network is given in Fig. 2, where the news topics are connected and organized according to the strength of their associations, $\Upsilon(\cdot, \cdot)$. One can observe that such topic network can provide a good global overview of large-scale collections of news videos at the topic level and can precisely characterize the interestingness of the relevant news topics, thus it can be used to assist users on making better search decisions.

To integrate the topic network for supporting personalized topic recommendation, it is very attractive to achieve graphical representation and visualization of the topic network, so that the users can obtain a good global overview of large-scale collections of news videos at the first glance and make better search decisions in the interactive topic network exploration and navigation process. Thus the underlying topic network visualization techniques should be able to provide a good balance between the local detail and the global context. The local detail is used to help users focus on the news topics of interest in their current focus. The global context is needed to tell the users where the other relevant news topics are (i.e., which news topics they can search for next step) and their contextual relationships with the news topic in the current focus, such global context can suggest the new search directions effectively. Thus supporting visualization and interactive navigation of the topic network is becoming a complementary and necessary component for personalized news video recommendation system and it may lead to the discovery of unexpected news videos and guide the future search directions effectively.

Unfortunately, visualizing large-scale topic network in a 2D system interface with a limited screen size is not a trivial task. To achieve more effective visualization of largescale topic network, we have developed multiple innovative techniques: (a) highlighting 
the news topics according to their interestingness scores for allowing users to obtain the most important insights at the first glance; (b) integrating hyperbolic geometry to create more space for large-scale topic network visualization and reduce the potential overlappings via interactive exploration.

We have integrated both the popularity of the news topics and the importance of the news topics to determine their interestingness scores. The popularity of a given news topic is related to both the number of TV news programs which have discussed the given news topic and the time length for the same TV news program to report the given news topic. If one news topic is discussed by more TV news programs simultaneously or reported by the same TV news program repeatedly for a long time, it tends to be more interesting in general. The importance of a given news topic is related to its linkage structure with other news topics on the topic network. If one news topic is related to more news topics on the topic network, it tends to be more interesting. For example, the news topic for "roadside bond in Iraq" may strongly relate to the news topics of "gas price increase" and "stock price decrease". Thus the interestingness score $\rho\left(C_{i}\right)$ for a given news topic $C_{i}$ is defined as:

$$
\rho\left(C_{i}\right)=\lambda \cdot \frac{e^{m\left(c_{i}\right)}-e^{-m\left(c_{i}\right)}}{e^{m\left(c_{i}\right)}+e^{-m\left(c_{i}\right)}}+\eta \cdot \frac{e^{t\left(c_{i}\right)}-e^{-t\left(c_{i}\right)}}{e^{t\left(c_{i}\right)}+e^{-t\left(c_{i}\right)}}+\gamma \cdot \frac{e^{k\left(c_{i}\right)}-e^{-k\left(c_{i}\right)}}{e^{k\left(c_{i}\right)}+e^{-k\left(c_{i}\right)}}
$$

where $\lambda+\eta+\gamma=1, m\left(c_{i}\right)$ is the number of TV news programs which have discussed or reported the given news topic $C_{i}$ simultaneously, $t\left(c_{i}\right)$ is the time length (days) for the same TV news program to report the given news topic $C_{i}$ repeatedly, $k\left(c_{i}\right)$ is the number of news topics linked with the given news topic $C_{i}$ on the topic network. Such interestingness scores can be used to highlight the most interesting news topics and eliminate the less interesting news topics for reducing the visual complexity for largescale topic network visualization and exploration.

Supporting graphical representation and visualization of the topic network can provide an effective solution for exploring large-scale collections of news videos at the topic level and recommending the news topics of interest to the users interactively for assisting them to make better search decisions. However, visualizing large-scale topic network in a $2 \mathrm{D}$ system interface with a limited screen size is a challenging task. We have investigated multiple solutions to tackle this challenge task: (a) A string-based approach is incorporated to visualize the topic network with a nested view, where each news topic node is displayed closely with the most relevant news topic nodes according to the values of their associations. The underlying inter-topic contexts are represented as the linkage strings. (b) The geometric closeness of the news topic nodes is related to the strength of their inter-topic contexts, so that such graphical representation of the topic network can reveal a great deal about how these news topics are connected. (c) Both geometric zooming and semantic zooming are integrated to adjust the levels of visible details automatically according to the discerning constraint on the number of news topic nodes that can be displayed per view.

Our approach for topic network visualization has exploited hyperbolic geometry [8]. The hyperbolic geometry is particularly well suited for achieving graph-based layout of the topic network, and it has "more space" than Euclidean geometry. The essence of our approach is to project the topic network onto a hyperbolic plane according to the inter-topic contexts, and layout the topic network by mapping the relevant news topic nodes onto a circular display region. Thus our topic network visualization scheme takes 
the following steps: (a) The news topic nodes on the topic network are projected onto a hyperbolic plane according to their inter-topic contexts by performing curvilinear component analysis (CCA), and such CCA process can be obtained automatically by preserving the local inter-topic contexts between the neighboring news topic nodes on the topic network, e.g., through minimizing the following error function:

$$
E\left(\left\{C_{i}\right\}\right)=\frac{1}{2} \sum_{i=1}^{n} \sum_{j>i}^{n} \sigma_{i j}\left|\delta\left(C_{i}, C_{j}\right)-\Upsilon\left(C_{i}, C_{j}\right)\right|^{2}
$$

where $\Upsilon\left(C_{i}, C_{j}\right)$ is the strength of the inter-topic context between the news topics $C_{i}$ and $C_{j}$, the weight factors $\sigma_{i j}$ are chosen as a bounded and monotically descreasing function to allow the CCA projection algorithm to preserve the strong inter-topic contexts than of weak inter-topic contexts (i.e., preserving the contexts between neighboring news topic nodes on the topic network), and $\delta\left(C_{i}, C_{j}\right)$ is the location distance between the news topics $C_{i}$ and $C_{j}$ on the hyperbolic plane.

$$
\delta\left(C_{i}, C_{j}\right)=2 \cdot \operatorname{arctanh}\left(\frac{\left|X_{c_{i}}-X_{c_{j}}\right|}{\left|1-X_{c_{i}} \bar{X}_{c_{j}}\right|}\right)
$$

where $X_{c_{i}}$ and $X_{c_{j}}$ are the physical locations of the news topics on the hyperbolic plane. In our current experiments, the weigh factors $\sigma_{i j}$ are characterized by using a sigmoid function:

$$
\sigma_{i j}=\frac{e^{\Upsilon\left(C_{i}, C_{j}\right)}}{e^{\Upsilon\left(C_{i}, C_{j}\right)}+1}
$$

Through CCA projection, our algorithm can precisely preserve the local inter-topic contexts, and the global geometry is also preserved effectively because the nearest neighborhoods for the neighboring news topics are overlapped. (b) After such contextpreserving projection of the news topic nodes is obtained, Poincare disk model [8] is used to map the news topic nodes on the hyperbolic plane onto a 2D display coordinate. Poincaré disk model maps the entire hyperbolic space onto an open unit circle, and produces a non-uniform mapping of the news topic nodes to the 2D display coordinate.

\section{Personalized News Video Recommendation}

Because the same news topic may be reported many times in the same TV news program or be discussed simultaneously by many TV news programs for different broadcast stations, the amount of the news videos under the same news topic could be very large. Thus topic-based news search via simplely keyword matching may return large amount of news videos which are relevant to the same news topic. To reduce user's information overload, it is very important to develop new algorithms for ranking the news videos under the same news topic and recommending the most relevant news videos according to their importance and representiveness scores.

The news videos, which are relevant to the given news topic $C_{j}$, are ranked according to their importance and representiveness scores. For the given news topic $C_{j}$, the importance and representativeness score $\varrho\left(x \mid C_{j}\right)$ for one particular news video $x$ is defined as:

$$
\varrho\left(x \mid C_{j}\right)=\epsilon e^{-\Delta t}+(1-\epsilon) \frac{e^{\sigma\left(x \mid C_{j}\right)}-e^{-\sigma\left(x \mid C_{j}\right)}}{e^{\sigma\left(x \mid C_{j}\right)}+e^{-\sigma\left(x \mid C_{j}\right)}}
$$




$$
\sigma\left(x_{j}\right)=\zeta \cdot v\left(x \mid C_{j}\right)+\nu \cdot r\left(x \mid C_{j}\right)+\varsigma \cdot q\left(x \mid C_{j}\right)
$$

where $\zeta+\nu+\varsigma=1, \Delta t$ is the time difference between the broadcast time for the given news video $x$ for the particular TV news program and the time for the user to submit their requests, $v\left(x \mid C_{j}\right)$ is the visiting times for the given news video $x$ from all the users, $r\left(x \mid C_{j}\right)$ is the rating score of the given news video $x$ from all the users, $q\left(x \mid C_{j}\right)$ is the quality of the given news video.

We separate the time factor from other factors for news video ranking because the time factor is more critical than other factors for news video ranking (i.e., one topic can be treated as the news because it is new and tell people what is happening recently or what is discussing recently) and most people may just want to know the most recently reports for the given news topic. The quality $q\left(x \mid C_{j}\right)$ is simply defined as the frame resolution and the length of the given news video $x$. If a news video has higher frame resolution and longer length (be discussed for longer time), it should be more important and representative for the given news topic.

After the users' search goals (i.e., which are represented by the accessed news topics) are captured interactively, our personalized news video recommendation system can: (a) recommend top 5 news videos according to their importance and representativeness scores; (b) recommend other news topics of interest on the topic network which are most relevant to the accessed news topic and suggest them as the future search directions according to the user's current preferences, where the accessed news topic is set as the current focus (i.e., the center of the topic network); (c) recommend the most relevant online web news which are relevant with the accessed news topic, so that the user can also read the most relevant online web news; (d) record the user's search history and preferences for generating more reliable personalized topic network to make better recommendation in the future. Some experimental results are given in Fig. 3, and one can conclude that our personalized news video recommendation system can effectively support multi-modal news recommendation from large-scale collections of news videos.

\section{Algorithm Evaluation}

We carry out our experimental studies by using large-scale collections of news videos (3 TV news channels captured $24 \times 7$ for more than 3 months). The topic network which consists of 4000 most popular news topics is learned automatically from large-scale collections of news videos. Our work on algorithm evaluation focus on: (1) evaluating the performance of our news topic detection algorithm and assessing the advantages for integrating multi-modal information channels for news topic detection; (2) evaluating the response time for supporting change of focus in our system, which is critical for supporting interactive navigation and exploration of large-scale topic network to enable user-adaptive topic recommendation; (3) evaluating the performance (efficiency and accuracy) of our system for allowing users to look for some particular news videos of interest (i.e., personalized news video recommendation);

Automatic news topic detection plays an important role in our personalized news video recommendation system. Based on this observation, our algorithm evaluation for our automatic news topic detection algorithm focuses on comparing its performance 


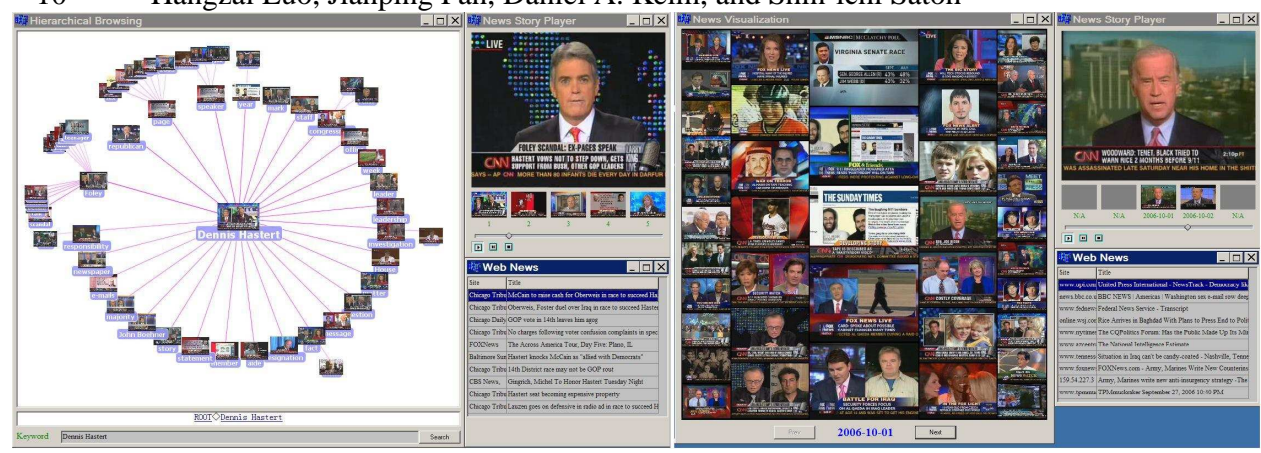

Fig. 3. Two examples for supporting personalized multi-modal news recommendation.

difference by combining different information channels for automatic news topic detection. We have compared three combination scenarios for news topic detection: (a) only the closed captions are used for news topic detection; (b) the closed captions and the audio channel are integrated and synchronized for news topic detection; (c) the closed captions, the audio channel and the video channel are seamlessly integrated and synchronized for news topic detection. As shown in Fig. 4, integrating multi-modal information channels (cross-media) for news topic detection can enhance the performance of our algorithm significantly.

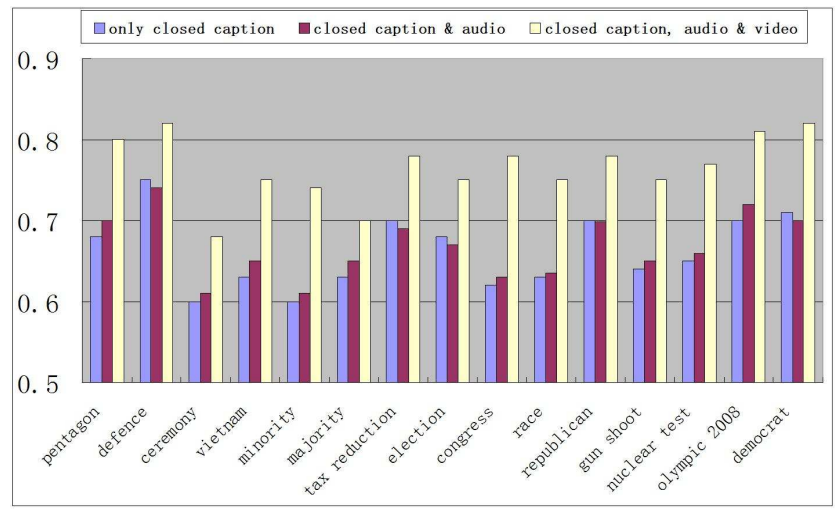

Fig. 4. The comparision results of our automatic news topic detection algorithm by integrating different information channels.

One critical issue for evaluating our personalized news video recommendation system is the response time for supporting change of focus to enable interactive topic network navigation and exploration, which is critical for supporting user-adaptive topic recommendation. In our system, the change of focus is used for achieving interactive exploration and navigation of large-scale topic network. The change of focus is implemented by changing the Poincaré mapping of the news topic nodes from the hyperbolic plane to the display unit disk, and the positions of the news topic nodes in the hyerbolic plane need not to be altered during the focus manipulation. Thus the response time for supporting change of focus depends on two components: (a) The computational time $T_{1}$ for re-calculating the new Poincaré mapping of large-scale topic network from a 
hyperbolic plane to a 2D display unit disk, i.e., re-calculating the Poincaré position for each news topic node; (b) The visualization time $T_{2}$ for re-layouting and re-visualizing the new Poincaré mapping of large-scale topic network on the display disk unit.

Because the computational time $T_{1}$ may depend on the number of news topic nodes, we have tested the performance differences for our system to re-calculate the Poincaré mappings for different numbers of news topic nodes. Thus our topic network with 4000 news topic nodes is partitioned into 5 different scales: 500 nodes, 1000 nodes, 2000 nodes, 3000 nodes, 3500 nodes and 4000 nodes. We have tested the computational time $T_{1}$ for re-calculating the Poincaré mappings of different numbers of news topic nodes when the focus is changed. From our experiments, we find that the computational time $T_{1}$ and the visualization time $T_{2}$ are not sensitive to the number of news topics, and thus re-calculating the Poincaré mapping and re-visualization for large-scale topic network can almost be achieved in real time. Thus our system can support change of focus in real time and achieve interactive navigation and exploration of large-scale topic network effectively.

For a given news topic of interest (news topic that is accessed by the particular user interactively), our system can further allow users to look for the most relevant news videos for the given news topic according to their importance and representative scores. To evaluate the effeciency and the accuracy of our personalized news video recommendation system, the benchmark metric includes precision $P$ and recall $R$. The precision $P$ is used to characterize the accuracy of our system for finding the most relevant news videos according to their importance and representativeness scores for the given news topic, and the recall $R$ is used to characterize the efficiency of our system for finding the most relevant news videos according to their importance and representativeness scores for the given news topic.

Table 1 gives the precision and recall of our personalized news video recommendation system. From these experimental results, one can observe that our system can support personalized news video recommendation effectively, thus users are allowed to obtain the most relevant news videos according to their importance and representativeness scores for the requested news topic.

\section{Conclusions}

A novel framework is developed to support personalized news video recommendation. To allow users to obtain a good global overview of large-scale collections of news videos at the topic level, topic network and hyperbolic visualization are seamlessly integrated to achieve user-adaptive topic recommendation. Thus users can obtain the news topics of interest interactively, build up their mental models of news needs easily and make better search decisions by selecting the visible news topics directly. Our system can further recommend the relevant web news, the new search directions, and the most relevant news videos according to their importance and representativeness scores. Our experiments on large-scale collections of news videos have provided positive results.

Acknowledgment

This work is supported by National Science Foundation under 0601542-IIS and 0208539-

IIS. Hangzai Luo is recently supported by Shanghai Pujiang Program under 08PJ1404600, NSFC 60496325 and 60803077, CSM 07dz5997, 863 R \& D 2006AA010111. 
Table 1. The precision and recall for supporting personalized news video recommendation.

\begin{tabular}{cccccc}
\hline news topics & policy & pentagon & change & dennis hastert & matter \\
\hline P/R & $95.6 \% / 97.3 \%$ & $98.5 \% / 98.9 \%$ & $100 \% / 99.2 \%$ & $95.3 \% / 88.3 \%$ & $85.2 \% / 85.3 \%$ \\
\hline news topics & implant & wedding & haggard & scandal & ethic \\
\hline P/R & $90.2 \% / 93.5 \%$ & $96.3 \% / 94.5 \%$ & $96.5 \% / 92.8 \%$ & $96.6 \% / 97.3 \%$ & $93.3 \% / 95.6 \%$ \\
\hline news topics & gate & steny hoyer & democrat & safety & investigation \\
\hline P/R & $95.9 \% / 96.8 \%$ & $96.5 \% / 96.2 \%$ & $96.3 \% / 97.1 \%$ & $94.5 \% / 94.8 \%$ & $93.3 \% / 96.5 \%$ \\
\hline news topics & majority & leader & confirmation & child & tax reduction \\
\hline P/R & $99.2 \% / 98.6 \%$ & $93.8 \% / 99.3 \%$ & $94.5 \% / 93.8 \%$ & $91.3 \% / 91.5 \%$ & $98.5 \% / 96.9 \%$ \\
\hline news topics & secretary & veterm & ceremony & beijing 2008 & program \\
\hline P/R & $100 \% / 98.8 \%$ & $99.8 \% / 99.2 \%$ & $99.3 \% / 96.6 \%$ & $99.2 \% / 97.3 \%$ & $83.5 \% / 90.2 \%$ \\
\hline news topics & honor & vietnam & lesson & teacher & conduct \\
\hline P/R & $91.2 \% / 93.5 \%$ & $98.8 \% / 96.7 \%$ & $90.3 \% / 91.6 \%$ & $93.8 \% / 94.5 \%$ & $87.92 \% / 88.3 \%$ \\
\hline news topics & minority & indonesia & president & republican & amish \\
P/R & $100 \% / 99.6 \%$ & $96.8 \% / 97.7 \%$ & $100 \% / 96.8 \%$ & $91.6 \% / 92.8 \%$ & $99.5 \% / 91.6 \%$ \\
\hline news topics & o.j. sinpson & trial & money & nuclear test & china \\
P/R & $95.6 \% / 99.4 \%$ & $90.5 \% / 90.3 \%$ & $100 \% / 90.6 \%$ & $100 \% / 97.6 \%$ & $97.3 \% / 95.2 \%$ \\
\hline news topics & john kerry & military & race & north korea & japan \\
\hline P/R & $100 \% / 96.5 \%$ & $100 \% / 93.2 \%$ & $100 \% / 97.8 \%$ & $100 \% / 99.3 \%$ & $98.5 \% / 95.6 \%$ \\
\hline news topics & election & leadship & school gun shoot & sex & message \\
\hline P/R & $100 \% / 95.5 \%$ & $92.8 \% / 90.3 \%$ & $100 \% / 96.7 \%$ & $97.5 \% / 98.2 \%$ & $88.3 \% / 87.6 \%$ \\
\hline \hline
\end{tabular}

\section{References}

1. G. Marchionini, Information seeking in electronic environments, Cambridge University Press, 1997.

2. B. Yang, T. Mei, X.-S. Hua, L. Yang, S.-Q. Yang, M. Li, "Online video recommendation based on multimodal fusion and relevance feedback", ACM CIVR'07, pp.73-80, 2007.

3. J.A. Wise, J. Thomas, K. Pennock, D. Lantrip, M. Pottier, A. Schur, V. Crow, "Visualizing the non-visual: Spatial analysis and interaction with information from text documents", IEEE InfoVis'95, pp.51-58, 1995.

4. R.C. Swan, J. Allan, "TimeMine: visualizing automatically constructed timelines", ACM SIGIR, 2000.

5. M. Weskamp, "Newsmap", http://www.marumushi.com/newsmap/index.cfm.

6. S. Havre, B. Hetzler, P. Whitney, L. Nowell, "ThemeRiver: Visualizing thematic changes in large document collections", IEEE Trans. on Visualization and Computer Graphics, vol.8, no.1, pp.9-20, 2002.

7. H. Luo, J. Fan, J. Yang, W. Ribarsky, S. Satoh, "Large-scale new video classification and hyperbolic visualization", IEEE VAST’07, pp.107-114, 2007.

8. H. Luo, J. Fan, J. Yang, W. Ribarsky, S. Satoh, "Exploring large-scale video news via interactive visualization", IEEE VAST'06, pp. 75-82, 2006.

9. W. Lai, X.-S. Hua, W.-Y. Ma, "Towards content-based relevance ranking for video search", ACM Multimedia, pp.627-630, 2006.

10. J. Teevan, S. Dumais, E. Horvitz, "Personalized search via automated analysis of interests and activities", ACM SIGIR, 2005.

11. H. Wactlar, A. Hauptmann, Y. Gong, M. Christel, "Lessons learned from the creation and deployment of a terabyte digital video library", IEEE Computer, vol.32, no.2, pp.66-73, 1999.

12. M.G. Christel, R. Yang, "Merging stryboard strategies and automatic retrieval for improving interactive video search", ACM CIVR'07, 2007. 